\title{
A Virtualization Desktop Application Platform of the Virtualized Graphics Processing Unit Technology on FlexPod Architecture
}

\author{
Shardrom Johnson ${ }^{1,2}$, Jun Zheng ${ }^{2,3}$ and Guorui Li $i^{1,4}$ \\ ${ }^{1}$ XianDa College of Economics and Humanities, Shanghai International Studies University, Shanghai 200083, Peoples Republic of China \\ ${ }^{2}$ School of Computer Engineering and Science, Shanghai University, Shanghai 200444, Peoples Republic of China \\ ${ }^{3}$ Informatization Office, Shanghai Institute of Visual Arts, Shanghai 201620, Peoples Republic of China \\ ${ }^{4}$ College of Information Science and Technology, Hunan Agricultural University, Changsha 410128, Peoples Republic of China
}

\begin{abstract}
Intelligent equipment and network application based on Artificial Intelligence, Big Data, Internet of Things and other emerging technologies are becoming one of the most important efforts in the construction of experimental and training information infrastructure in Colleges and Universities. Aiming at the problem that the application environment of teaching experiment and training in Colleges and Universities of Art and Design requires higher performance ability of virtual desktop and graphics processing, the specific characteristics on training room of art and design, and the design ideas and principles of virtualization desktop technology, are analysed on the unique perspective of specific projects of Shanghai Institute of Visual Arts. A virtualization desktop application platform of the Virtualized Graphics Processing Unit Technology on FlexPod Architecture is put forward. With the application of this platform, the performance of the desktop is improving, and the complexity of IT management is reducing, and the model for the application of the virtual desktop technology for colleges and universities of art and design is provided.
\end{abstract}

\section{Introduction}

Intelligent equipment and network application based on Artificial Intelligence, Big Data, Internet of Things and other emerging technologies are becoming one of the most important efforts in the construction of experimental and training information infrastructure in Colleges and Universities [1-2]. With the implementation of Outline of Reform and Development of China National Medium and Long-Term Education (2010-2020), virtualized desktop technology, one of the important contents of "accelerating the construction of educational information infrastructure", is integrated into the overall strategy of national informatization development [3-4]. Therefore, it has become one of the key tasks of promoting the educational information infrastructure in colleges and universities to actively carry out the application and practice of virtualized desktop technology [5-6].

\section{Specific characteristics on training room of art and design}

Colleges and universities have increasingly demanded applications of computer, and it appears diverse teaching needs. The decentralized management mode, where computing and storage functions in traditional teaching rooms, studios, libraries, etc. were directly completed by the terminal PC (Personal Computer) equipment, was obviously difficult to adapt to such application and teaching needs [7-8]. The complexity of application scenarios and the diversity of teaching requirements have made computer management more difficult, and the traditional computer-room management mode is difficult to meet such requirements [9-10]. The main performances are as follows: the frequency of the update of computer systems increases while the utilization rate does not improve; the update rates of application system may be significantly improved while the stability and safety are enormously reduced; the standardization of the overall IT equipment becomes more difficult while the workload of IT administrators is also significantly increased.

Although a large number of graphics workstations provide teachers and students with the convenience of teaching and learning in the early days, the deployment mode of traditional graphics workstations will still bring many problems over time, including: the declining performance of the machine year by year, the difficulty of management, and the increase of maintenance costs. Therefore, design colleges hope to find an efficient and flexible solution to reduce the maintenance costs of graphics workstation construction, and to make some adjustments to the resources and performance of graphics in the teaching mode of production, education and research.

The application and teaching needs of computer at design colleges are more urgent than those of ordinary universities, especially the requirements for computer graphics processing capabilities according to teaching. 
Currently, most of the lectures and student-learning are based on various graphics software with powerful graphics processing capabilities [7].

In this process of exploration, design colleges face two major challenges: Firstly, the update speed of teaching application must keep up with the pace of professional development; the IT resources needed in the teaching process can be supplemented in time, able to quickly provide a variety of practical and effective courseware, and supporting students to learn independently. secondly, the overall IT construction costs and maintenance costs should be reduced, avoiding the reinvestment of resources caused by replacement of hardware equipment and update of software system.

Shanghai Institute of Visual Arts, one of the members of the International Association of Art Design and Media Colleges and the only comprehensive visual arts college in Shanghai, whose school motto is "Fidelity, Excellence, Innovation, and Harmony", is committed to leading the visual concept of big vision, building a platform for creative visual art, and cultivating artistic talents. It constantly explores a school-running mode that is close to industry and society, and a talent-training mode for industry-university cooperation and resource sharing. Therefore, Shanghai Institute of Visual Arts urgently needs to build four media studios with three-dimensional graphics design capacity through the application of virtualized desktop technology. The specific construction standards include:

- Realizing the deployment of 124 virtualized graphics workstations; supporting 3dMax, MAYA, CATIA, etc.; having high computing and graphics processing capability.

- Supporting professional graphics cards; able to implement GPU (Graphics Processing Unit) virtualization (vGPU), with each vGPU performance not lower than the current graphics performance of the new graphics workstations in the university.

- Needing to have independent virtual desktops that are under centralized management for teachers and students.

- Providing smooth high-definition video by virtual desktops, multimedia communication via instant messaging software, smooth viewing of web pages with Flash, and the use of a variety of peripherals.

- Refining network and traffic awareness to achieve intelligent acceleration of desktop delivery; reducing bandwidth consumption by applying advanced compression and deduplication to the data.

- Redirecting Flash multimedia over the network with a 300 millisecond delay, ensuring a local-user experience even on mobile networks or in very remote locations.

- Providing clear voice and video with low bandwidth requirements; supporting users' various communication and collaboration; using high-quality multimedia to help teachers and students to teach and learn economically, efficiently and creatively.

- Rendering multimedia content with the processing of endpoint devices.

\section{Design ideas and principles of virtualization desktop technology}

The Virtualized Desktop Technology Application Project of the Shanghai Institute of Visual Arts will deploy and integrate the Virtual Desktop Infrastructure (VDI) environment based on its cloud computing platform built in 2013. It can utilize the interconnected matrix switch in the existing UCS (Unified Computing System) and the Cisco server in the new platform to interconnect with the FCoE (Fiber Channel over Ethernet) to implement unified management of the new rack server platform and the existing blade system.

\subsection{Design ideas}

In the physical architecture construction, the virtualized desktop technology application project of Shanghai Institute of Visual Art fully considers the security, reliability and scalability of the platform. In the network area, redundant mode is deployed using existing equipment to ensure network connectivity. In the server area, the Cluster in the virtualization platform is utilized to achieve high availability of the host, and two existing UCS interconnected matrix switches are used as the fiber switch to realize the link redundancy between the server and the storage device. In terms of storage, a single storage configuration with dual controller is deployed to ensure data security and reliability. In the meantime, the overall architecture platform adopts the full link redundancy deployment mode to ensure business continuity. In terms of management, unified application, unified management, unified resource allocation, and unified desktop publishing are implemented through the virtualization management platform to achieve security, manageability, reliability, and scalability of the entire architecture platform.

Meanwhile, the Virtualized Desktop Technology Application Project of Shanghai Institute of Visual Arts will be based on the teaching of "Uniform Standards, Unified User Management, Unified Construction Platform, and Unified Database" to facilitate data sharing and application, which is conducive to data search and analysis. Under the premise of security and authority, users can legally access the storage resources in the architecture platform through unified-domain user identity authentication, and thus a sustainable development platform will be constructed based on unified information, unified identity authentication, and unified data centre.

\subsection{Design principles}

Under the guidance of "unified planning, step-by-step implementation, enhanced application, integration of resources, and sharing of data", the virtualized desktop technology application project of Shanghai Institute of Visual Arts adopt "reliability, security, scalability, operationality and manageability" as its design principles, takes account of the advancement and maturity of technology, and adopts a modular design approach. 


\subsubsection{Unified planning}

Virtualized desktop technology application project involves computer technology, network technology, communication technology, storage technology and network engineering, software engineering, project management, etc., featuring high investment, long cycle, and multiple applications. Therefore, before construction, we must have a holistic view, do a good job in project analysis, planning and design to ensure unified information standards, technical route, infrastructure and management.

\subsubsection{Step-by-step implementation}

Virtualized desktop technology application project involves requirements research, solution demonstration, system selection, deployment and integration, personnel training, promotion and application, operational feedback, modification and improvement, etc. Therefore, the entire construction process must be coordinated and implemented step by step to ensure the progress and quality of the project and reduce the risk of failure.

\subsubsection{Strengthening application}

Application, the core of the virtualized desktop technology application project, enables various functional departments to realize informationization of management and teaching, simpler and faster communication between different departments, and data sharing and exchange between different functional departments, improves the scientific and democratic decision-making, increases efficiency by reducing staff, form a dynamic new management mechanism, and provides personalized and comprehensive information services for teachers and students.

\subsubsection{Integration of resources}

The virtualized desktop technology application project needs to face various application systems purchased and developed by various educational institutions after years of informatization construction, and a large amount of information resources deposited by them. Considering the protection of existing investments, making full use of existing information resources, new application projects must continuously integrate existing information resources, develop new resources, and build centralized information management mechanism.

\subsubsection{Sharing data}

The virtualized desktop technology application project must take into account that education is service-oriented, ensuring mutual access of data between multiple legacy application systems, and ensuring data sharing and realtime exchange between application systems.

\section{Virtualized graphics processing unit technology on FlexPod architecture}

The overall platform architecture of virtualized desktop technology is achieved by using the cloud computing platform built in 2013 and a proven FlexPod architecture based on the Cisco UCS Unified Computing System, Nexus Data Center Switch and Netapp FAS Unified Storage (as Figure 1 shown), deploying Citrix Xendesktop desktop virtualization on the FlexPod architecture and realizing the release of the final virtualized graphics workstation in conjunction with the Nvidia GRID K2 graphics card (as Figure 2 shown).

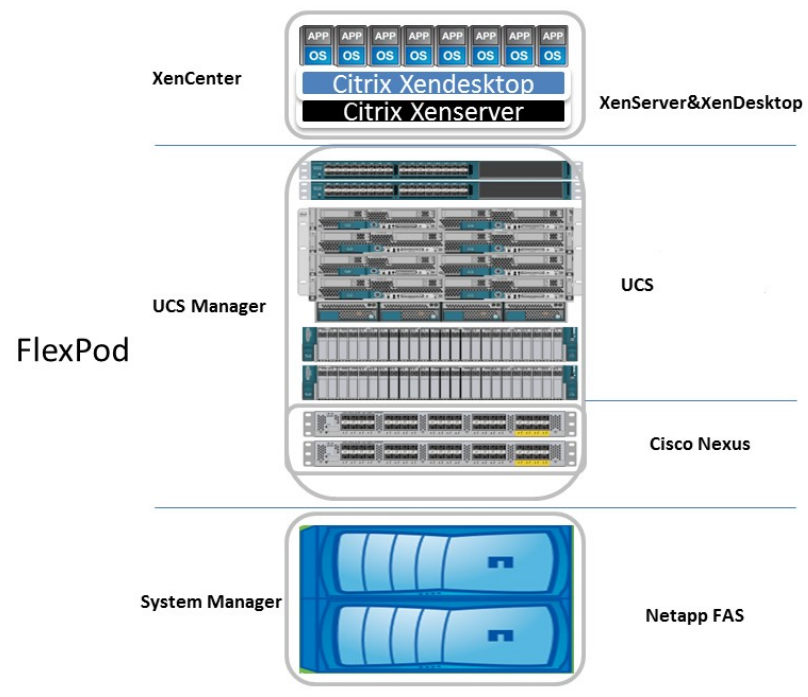

Figure 1. FlexPod architecture.

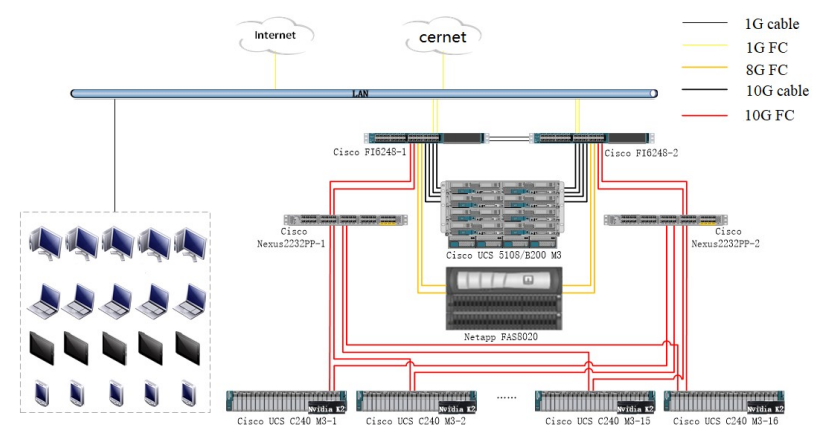

Figure 2. Architecture of virtualized desktop technology.

In terms of the server, 16 Cisco UCS C Series highperformance rack servers and a new generation of Nvidia VGX K2 high-performance graphics cards are used to meet the memory requirements of virtualized graphics workstations. By the Nexus 2232PP, 10G link can be performed using the Cisco Virtual Interface Card VIC with the UCS FI6248. Therefore, the 16 Cisco UCS C240 M3s in this project can be uniformly managed by the UCS Manager in UCS FI6248, and by a single management platform with the existing UCS blade server Moreover, UCS stateless computing is used to realize unified deployment of all Cisco UCS C240 M3 server information. 
With regard to the memory, Netapp's next-generation high-performance storage, dual controllers and $48 \mathrm{~Gb}$ cache are used, and Flash Cache technology are adopted through $4 * 200 \mathrm{~Gb}$ SSD hard disks to achieve performance improvement. What's more, the storage provides $16 \mathrm{~Gb}$ FC ports, which can be backward compatible with $8 \mathrm{~Gb} / 4 \mathrm{~Gb}$ FC. Since the project uses the unified port of UCS FI6248 as the storage connection port, and FI6248 can support up to $8 \mathrm{~Gb} \mathrm{FC}$, therefore, this project uses $8 \mathrm{~Gb} \mathrm{FC}$ to exchange data with the server in storage connection.

The virtualized graphics workstation platform adopts Citrix Xendesktop, the industry's leading desktop virtualization product, and delivers 3D application publishing in high-definition mode using Citrix HDX 3D Pro technology. The bottom layer supports Citrix XenServer and virtualizes physical GPU with Nvidia GRID K2 (as Figure 3 shown). The Nvidia VGX K2 card in this project supports virtual 8 vGPUs, so a single server of this project can carry up to 8 virtual graphics workstations, and a separate vGPU mode is assigned to each virtual desktop. Finally, a virtualized graphics workstation is realized by using the desktop virtualization model (as Figure 4 shown).

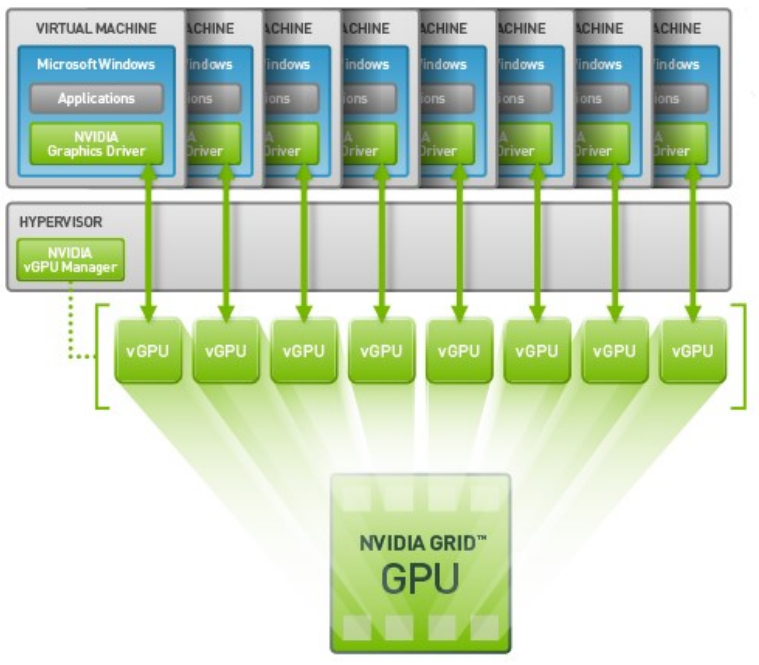

Figure 3. Virtualization of GPUs with Nvidia GRID K2.

\section{Advantages of our virtualized desktop application}

The Virtualized Desktop Technology Application Project of Shanghai Institute of Visual Arts uses a pre-configured and proven design that reduces risk and TCO (Total Cost of Ownership), providing balanced CPU, memory, I/O and storage capacity for optimal performance. The purpose of the project is fully achieved by the optimized performance that can quick clone and configure the desktop. The specific advantages are reflected in the following aspects:

Firstly, it is easy-to-deploy. Fully-featured virtual graphics workstations can be built by rack-mount servers; it can realize integrated backup, data protection, and disaster recovery and have continuous access to desktops, applications and data.
Secondly, in terms of deployment or scaling, rapid desktop cloning and configuration, as well as the flexibility are realized to achieve dynamic on-demand scaling.

Thirdly, by using the Cisco VIC virtual interface card in the desktop virtualization environment, 256 independent channel addresses can be virtualized, which fully meets the requirements of running 8 virtual graphics workstations on a single physical server of the project. Meanwhile, fine-grained management control is made possible by the address assigned to each virtual machine.

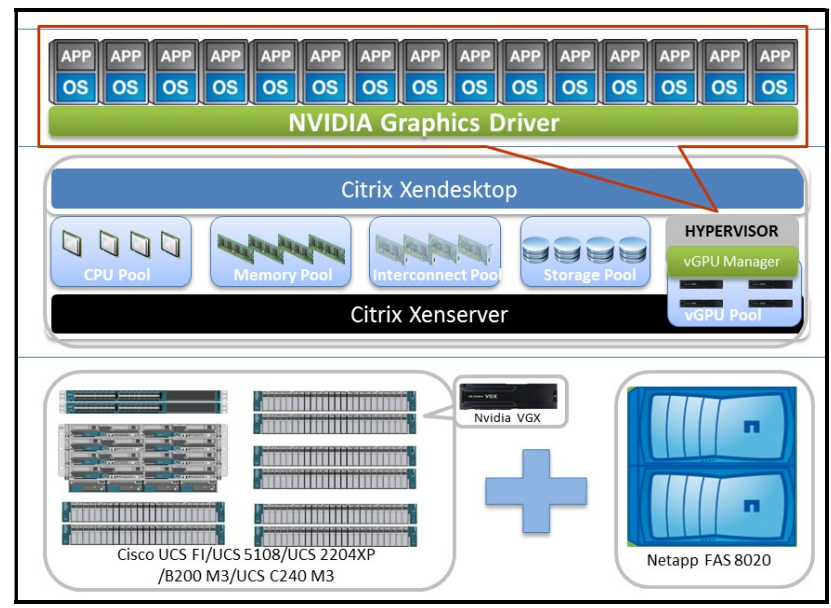

Figure 4. Virtualization Desktop Application Platform.

Fourthly, Netapp's data deduplication technology is used. In the case of multiple virtual desktops, and using the same operating system or application, turning on data deduplication can greatly reduce the storage space utilization rate and effectively protect the project investment.

At last, it can support complex 3D graphics applications, allowing 3D graphics applications to work on virtual desktops close to traditional graphics workstations; if combined with the new H.264 compression technology, it can also deliver various graphics design applications to any mobile device as needed.

\section{Conclusion}

Since its official operation in 2017, a virtualization desktop application platform of the Virtualized Graphics Processing Unit Technology on FlexPod Architecture of Shanghai Institute of Visual Arts has fully achieved its original design goals and performance. Now it has been able to provide up to 360 virtual graphics workstations at the same time, providing a high-quality user experience for all teachers and students on campus. In the later period, the application efficiency of virtualized desktops will be further expanded to better serve the specific needs of art-based private colleges and universities, and improve the construction of educational information infrastructure. 


\section{Acknowledgment}

The theory of this research work is supported by the National Natural Science Fund Project (61303097), the Shanghai Science Fund Project (17ZR1428400), the Second (2016) Shanghai Research Project for Private University (2016-SHNGE-08ZD), and the Shanghai University Graduate Innovation Fund Project (SHUCX120105) the support of this paper work.

\section{References}

1. X.S. Guo, L. Li, N. Ansari, B. Liao, "Knowledge Aided Adaptive Localization via Global Fusion Profile", IEEE Internet of Things Journal, 5 (2): 1081-1089, (2018), doi: 10.1109/JIOT.2017.2787594.

2. Z.H. Tian, Y. Cui, L. An, S. Su, X.X. Yin, L.H. Yin, X. Cui, "A Real-Time Correlation of Host-Level Events in Cyber Range Service for Smart Campus", IEEE Access, 6: 35355-35364, (2018), doi: 10.1109/ACCESS.2018.2846590.

3. D.F. He, Y.T. Pan, Z. Ma, J.T. Wang, "Sustainable growth in China's communications field: Trend analysis of impact of China's academic publications", China Communications, 10 (3): 157-163, (201), doi: 10.1109/CC.2013.6488844.

4. S.E. August, M.L. Hammers, D.B. Murphy, A. Neyer, P. Gueye, R.Q. Thames, "Virtual Engineering Sciences Learning Lab: Giving STEM Education a Second Life", IEEE Transaction on Learning Technologies, 9 (1): 18-30, (2016), doi: 10.1109/TLT.2015.2419253.
5. W.M. Kunkle, R.B. Allen, "The Impact of Different Teaching Approaches and Languages on Student Learning of Introductory Programming Concepts", ACM Transactions on Computing Education, 16 (1): 3-1-3-26, (2016), doi: 10.1145/2785807.

6. S. Goel, D. Pon, P. Bloniarz, R.B.-Drowns, G. Berg, V. Delio, L. Iwan, T. Hurbanek, S.P. Schuman, J. Gangolly, A. Baykal, J. Hobbs, "Innovative model for information assurance curriculum: A teaching hospital", Journal on Educational Resources in Computing, 6 (3): 2-1-2-15, (2006), doi: 10.1145/1243481.1243483.

7. M. Joy, B. Muzykantskii, S. Rawles, M. Evans, "An Infrastructure for Web-Based Computer-Assisted Learning", Journal on Educational Resources in Computing, 2 (4): 4-1-4-19, (2002), doi: 10.1145/949257.949261.

8. S.F. Fang, L.D. Xu, Y.Q. Zhu, J. Ahati, H. Pei, J.W. Yan, Z.H. Liu, "An Integrated System for Regional Environmental Monitoring and Management Based on Internet of Things", IEEE Transactions on Industrial Informatics, 10 (2): 1596-1605, (2014), doi: 10.1109/TII.2014.2302638.

9. Y.Y. Wang, "On the Application of Cloud Desktop Technology to the Management of Computer Rooms"(in Chinese), Journal of Jilin Teachers Institute of Engineering and Technology, 31 (10): 94-96, (2015), doi: 10.3969/j.issn.10099042.2015.10.032.

10. S. Johnson, D. Hsu, G.F. Wu, "The Application and Research of CSET"(in Chinese), Journal of Computer Applications and Software, 20 (5): 66-68, (2003), doi: 10.3969/j.issn.1000-386X.2003.05.027. 